\title{
Aberration-Corrected STEM/EELS at Cryogenic Temperatures
}

\author{
Lena F. Kourkoutis ${ }^{1,2}$, Ismail El Baggari ${ }^{3}$, Benjamin H. Savitzky ${ }^{3}$, David J. Baek ${ }^{4}$, Berit H. Goodge ${ }^{1}$, \\ Robert Hovden ${ }^{1}$, Michael J. Zachman ${ }^{1}$ \\ 1. School of Applied and Engineering Physics, Cornell University, Ithaca NY 14853, USA. \\ 2. Kavli Institute at Cornell, Cornell University, Ithaca NY 14853, USA. \\ 3. Department of Physics, Cornell University, Ithaca NY 14853, USA. \\ 4. School of Electrical and Computer Engineering, Cornell University, Ithaca, NY, USA.
}

Today's aberration-corrected scanning transmission electron microscopes (STEM) routinely focus highenergy electrons down to a spot smaller than $1 \AA$ in diameter to perform scattering experiments that allow us to study the atomic-scale structure of materials and devices. When combined with electron energy loss spectroscopy analysis of the inelastically scattered electrons, these narrow probes can also provide atomic-scale information about the composition and local electronic structure of bulk materials, defects and interfaces $[1,2]$.

Most efforts, so far, have focused on room temperature measurements where stable imaging with sub- $\AA$ resolution is routinely achievable. However, a range of materials including complex oxides and transition-metal dichalcogenides (TMDs) exhibit exotic functionalities below room temperature. Correlating the atomic level structure and electronic properties at cryogenic temperatures is therefore an important step in realizing the goal of understanding and controlling emergent phenomena in these materials. Charge density waves (CDWs) and the coupled periodic lattice distortions (PLD), for example, govern the electronic properties in many layered TMDs. In particular, $1 \mathrm{~T}-\mathrm{TaS}_{2}$ can undergo a phase transition from a conducting to an insulating state upon cooling as the PLD becomes commensurate with the crystal lattice. Using an uncorrected FEI Tecnai F20 equipped with cryo-blades we have directly imaged PLDs in thin exfoliated $1 \mathrm{~T}-\mathrm{TaS}_{2}$ using atomic resolution cryo-STEM [3].

Aberration-corrected cryo-STEM on a FEI Titan Themis allows imaging at sub- $\AA$ resolution and atomic tracking with picometer precision. This, however, requires acquisition optimization to account for stage drift and instabilities during imaging at cryogenic temperature. A series of rapid acquisitions with sufficiently large SNRs to allow for effective rigid registration of frames has proven to be successful demonstrating an information transfer below $1 \AA$ (Figure 1). Using this technique we have mapped the evolution of PLD stripes in $\mathrm{Bi}_{1-\mathrm{x}}(\mathrm{Sr}, \mathrm{Ca})_{\mathrm{x}} \mathrm{MnO}_{3}$, a model charge-ordered system with $\mathrm{T}_{\mathrm{c}} \sim 300 \mathrm{~K}$. We observe temperature-dependent inhomogeneities in the stipe order such as shear deformations and topological defects, and the emergence of phase coherence well below $\mathrm{T}_{\mathrm{c}}$. Atomic resolution spectroscopic mapping, which has more stringent stability requirements, is successfully performed using a direct electron detector (DED) - a Gatan K2 Summit operated in electron counting mode (Figure 2). The detector's superior SNR for low-dose applications enables spectroscopic mapping at reduced dwell times. Real space visualization of strongly correlated materials by aberration-corrected cryoSTEM/EELS promises a deeper understanding of these complex systems [4].

[1] D. A. Muller et al., Science 319 (2008), p. 1073.

[2] J. Mundy et al., Nat. Commun. 5 (2014), p. 3464.

[3] R. Hovden et al., Proc. Natl. Acad. Sci. U.S.A. 113 (2016), p. 11420. 
[4] We acknowledge support from AFOSR (FA 9550-16-1-0305), NSF MRI award (DMR-1429155), and the Packard Foundation. Work made use of CCMR facilities supported by NSF (DMR-1120296).
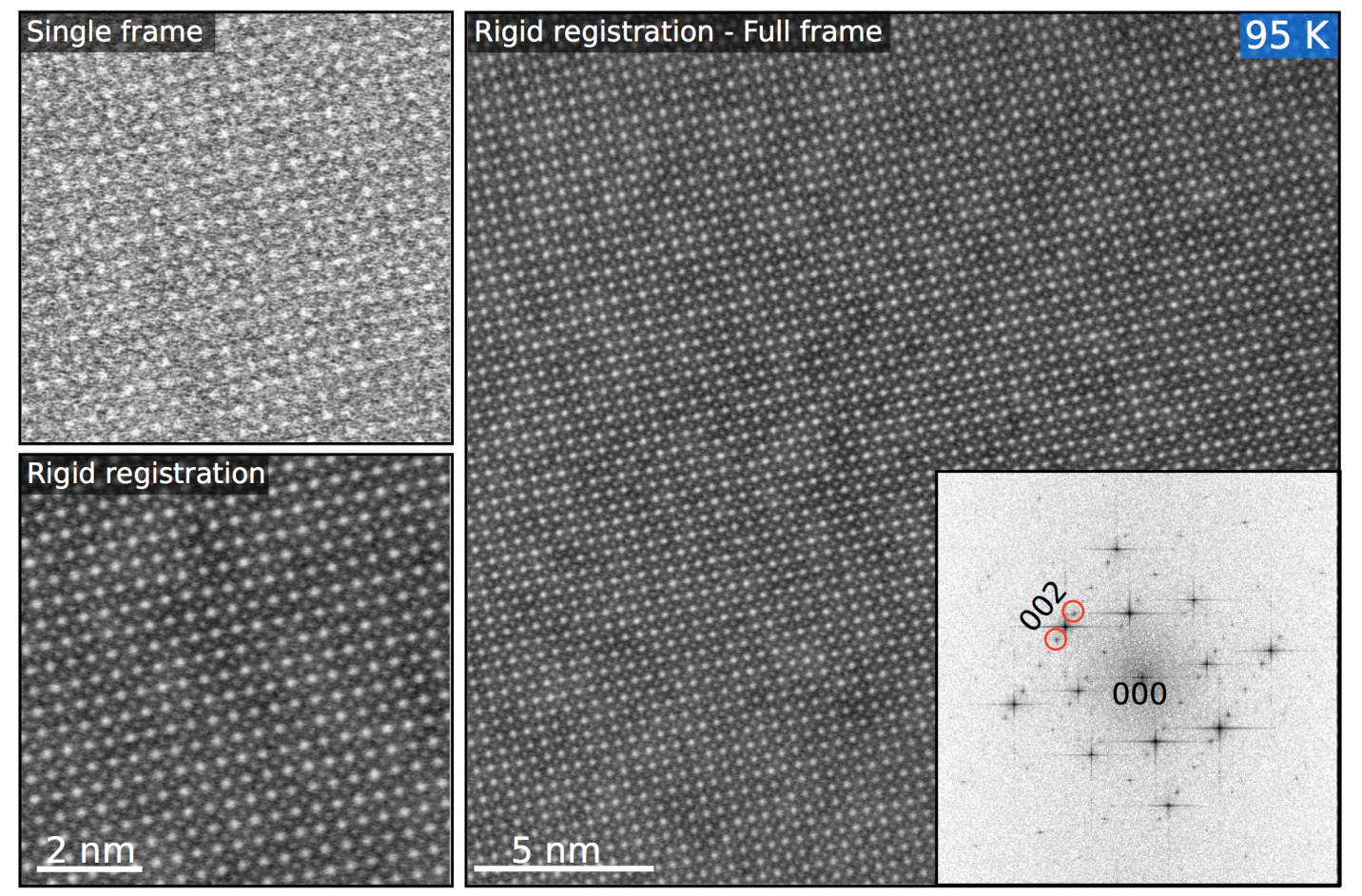

Figure 1. Aberration-corrected cryo-STEM of a stripe ordered manganite. (top left) Small section of a $2 \mathrm{kx} 2 \mathrm{k}$ single frame recorded at a dwell times of $0.5 \mathrm{~s} /$ pixel. (bottom left) Same section after rigid registration of all frames. (right) Large field of view HAADF cryo-STEM image (frame averaged) of the manganite oxide $\mathrm{Bi}_{1-\mathrm{x}}(\mathrm{Sr}, \mathrm{Ca})_{\mathrm{x}} \mathrm{MnO}_{3}$. The FFT shows PLD peaks (two marked with red circles) decorating the Bragg peaks.
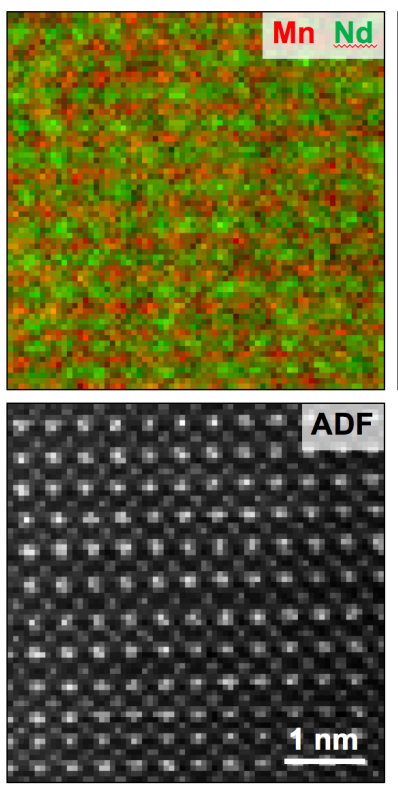
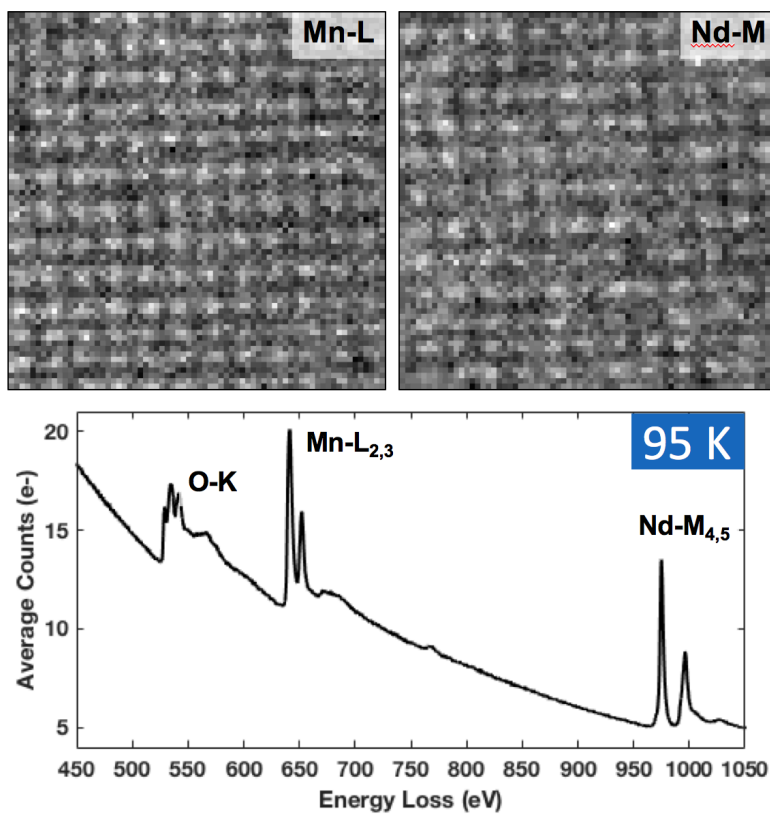

Figure 2. Atomic-resolution spectroscopic mapping of $\mathrm{Nd}_{0.5} \mathrm{Sr}_{0.5} \mathrm{MnO}_{3}$ at cryogenic temperature. The $70 \times 70$ pixel map was recorded in $\sim 13 \mathrm{~s}$ on a customized FEI Titan Themis with 965 GIF Quantum ER, K2 Summit in counting mode and a $\mathrm{LN}_{2}$ double-tilt cryo-holder. Compared to traditional indirect detectors, the low read-out noise ensures that even at short dwell times $(2.5 \mathrm{msec} / \mathrm{pixel})$ the SNR allows mapping of the $\mathrm{Mn}-\mathrm{L}$ and Nd-M edges. Rapid mapping enables spectroscopic studies at low temperatures. 